\author{
Jacopo Santoro* \\ Università degli Studi di Padova
}

\title{
MARIO PRAZ TRADUTTORE DEL CORVO DI EDGAR ALLAN POE
}

\begin{abstract}
L'articolo si pone l'obiettivo di analizzare dal punto di vista storico-linguistico e metrico-stilistico una delle prime traduzioni di Mario Praz dalla lingua inglese e di ricostruire un quadro generale riguardante il suo metodo di traduzione. Si è scelta la traduzione del Corvo di Edgar Allan Poe poiché in essa si vedono in embrione tutti i procedimenti traduttivi che andranno poi a comporre le linee guida che Praz sempre seguirà nelle proprie rese in lingua italiana. Scelte caratterizzate, come risulta da un'analisi dei testi critici nei quali l'autore riflette sulla sua teoria di traduzione, dal continuo tentativo di individuare e rendere nella lingua d'arrivo le dominanti che costituiscono le principali ragioni di composizione e la posizione culturale che il testo da tradurre ricopre nella lingua di partenza. Nel tradurre $\mathrm{Il}$ Corvo Praz decide perciò di mantenere quanto più possibile la forma metrica dell'originale, adattandola al sistema versificatorio italiano; inoltre ricalca il complicato schema rimico e le continue tessiture foniche del testo originale mentre dal punto di vista linguistico fa una serie di scelte volte a richiamare il linguaggio arcaizzante di Poe. Esito finale della traduzione è un testo che si avvicina all'atmosfera dell'originale, trasponendone insieme il fascino e alcuni limiti poetici.
\end{abstract}

Parole chiave: Praz, traduzione, Il Corvo, Poe, dominanti, stilistica.

\section{TEMPI E MOTIVI DELLA TRADUZIONE}

Nel 1921 Mario Praz affrontava una delle sue prime traduzioni, Il corvo $^{1}$, forse la più celebre poesia di Edgar Allan Poe. La scelta del venticinquenne critico romano non stupisce più di tanto, sia per la coerenza con i suoi interessi cronologici ${ }^{2}$ quanto con quelli tematici ed estetici3; inoltre entrambi gli autori percorrono il non certo troppo battuto ambito della

*jacopo.santoro@studenti.unipd.it

${ }^{1}$ Poe e Praz (1921).

${ }^{2}$ Nel 1925 uscirà infatti Poeti Inglesi dell'Ottocento; v. Praz (1925).

${ }^{3}$ Non a caso nel 1930 usciva la sua più famosa raccolta di saggi: La carne, la morte e il diavolo nella letteratura romantica. 
saggistica dell'arredamento d'interno ${ }^{4}$. Non a caso egli tornerà sui motivi della traduzione di questo testo in un breve saggio dove dirà:

Le versioni del Corvo quasi non si contano, da quella del povero Ernesto Ragazzoni a quella perpetrata dal sottoscritto che da giovane, come tutti i giovani, ebbe il suo momento d'infatuazione pel poeta che i critici anglosassoni [...] si sforzano, e si sforzano piuttosto invano, di farci considerare in una luce ben più modesta di quella fulgidissima della quale ci par di vederlo aureolato nel Parnaso dei sommi (Praz 2003: 144).

Come è facile vedere, il giudizio critico sul poeta americano è ambiguo: egli risulta agli occhi di un più adulto Praz sia poeta adatto a facili infatuazioni giovanili sia poeta in grado di difendere la somma gloria dei suoi versi dagli attacchi riduzionistici portati avanti dai critici anglosassoni. Questa ambiguità di giudizio è anche nella Postfazione (Poe e Praz 2012) al volume $\mathrm{Il}$ corvo ${ }^{5}$, dove Praz rimprovera a Poe l'eccessiva rozzezza dello stile, imputandogli di accontentarsi di raggiungere un facile effetto con immagini "approssimative e vistose" (Poe e Praz 2012: 113) e di comporre versi macchinosi a causa dell'eccessivo calcolo razionale; ma alla fine non riesce a negarne del tutto la suggestività ${ }^{6}$. La poesia di Poe per Praz ha dunque "lo stesso tipo di vitalità fissa, allucinata di molti dei suoi racconti: la pseudovitalità delle figure di cera" (Poe e Praz 2012: 113) e manca "di quel soffio indefinibile che è la vita stessa" (Poe e Praz 2012: 113); al contempo però Praz sceglie di tradurre $I l$ corvo, e di rendere il testo cercando quanto più possibile di rispettare le scelte stilistiche dell'autore americano; e fa ciò perché riconosce a Poe una grandezza che va oltre qualunque strumentazione tecnica di maniera, cioè la grandezza di un autore in grado di far trasudare dalle proprie opere la propria angoscia personale ${ }^{7}$. Non sarà forse azzardato dire che questa angoscia emerge così forte proprio perché Poe sembra eclissare, tanto in alcuni racconti quanto nel Corvo, la propria soggettività, mostrando nelle prose un intreccio sempre razionalmente inattaccabile e nella poesia la forma della tradizione spogliata di qualunque trasporto autoriale, costruendola a tavolino nei contenuti e nelle forme ${ }^{8}$;

${ }^{4}$ Poe scrive The Philosophy of furniture nel 1840 (cfr. Poe (2014)); per l'opera di Praz cfr. Praz (1964).

${ }^{5}$ Edizione il cui testo è preso a riferimento per questa analisi.

6 "E si vedrà perché un deliberato premer di pedale su simili elementi, debba produrre sul lettore ingenuo un effetto irresistibile" (Poe e Praz 2012: 113).

7 “" [...] che si chiami subconscio o parte subliminare e inconfessabile dell'anima, Poe ce ne ha fatto sentire per primo la presenza nelle sue opere" (Praz 2003: 148).

${ }^{8}$ Operazione che l'americano esplicita nel saggio Philosophy of composition uscito nel 1846, appena un anno dopo la prima comparsa del Corvo. 
operazione che ricorda l'ultimo Beethoven secondo Thomas Mann': il musicista e lo scrittore, eliminando il soggetto dalle loro opere con una forma matematicamente costruita, toglierebbero ad esse qualunque possibilità di comunicare una qualsivoglia scintilla vitale proveniente dall'autore, evocando nei fruitori un sentimento di morte.

Proprio la capacità di far emergere questa angoscia, unita all'attenzione quasi ossessiva per il significante e gli aspetti fonici - sia in poesia che in prosa -, garantirà all'americano enorme fama già presso alcuni poeti a lui contemporanei e della generazione successiva. Nella Philosophy of composition ${ }^{10}$ Poe infatti dichiara che scopo primo della poesia è ottenere un effetto sul lettore, e non tanto il pieno intendimento del testo poetico; e all'ottenere suddetto effetto devono concorrere tutti gli aspetti della poesia, partendo dalla scelta del tema per arrivare alla sua veste formale e fonico-ritmica, passando per le scelte lessicali e di immagini evocate. Quanto appena detto fa capire molto bene l'influenza su Baudelaire prima e sui simbolisti francesi poi che Poe dovette esercitare, al punto che il giovane Mallarmé lo poté ritenere un padre putativo per la sua poesia pura $^{11}$; ma Praz ridimensiona l'entusiasmo del francese faticando a vedere questi esiti poetici come già maturi nel Poe versificatore, sulla cui capacità stilistica il critico romano è, come abbiamo visto sopra, abbastanza scettico ${ }^{12}$.

Poe sarebbe dunque considerabile "un parente povero, sia pure; ma di quei poveri che poi lasciano milioni agli eredi" (Praz 1972: 94), e si capisce così come questo progenitore di una parte del Novecento letterario abbia richiamato l'attenzione estetica dell'Anglista.

${ }^{9}$ Il musicista tedesco nella sua ultima produzione infatti, secondo Mann, sarebbe riuscito nell'operazione di eclissare del tutto la propria soggettività dalla composizione, così da presentare la forma tradizionale spogliata di ogni apporto individuale. Ma questa spoliazione angoscia l'ascoltatore, perché la pura forma evoca con perturbante memoria il più grande rimosso collettivo dell'umanità, la morte. Cfr. Doctor Faustus, cap. VIII. (v. Mann 1947/2017).

${ }^{10}$ Pubblicata da Poe per la prima volta nel 1846 in Graham's American Monthly Magazine of Literature and Art; la traduzione italiana utilizzata è reperibile in Poe e Praz (2012).

${ }^{11}$ Per approfondire il rapporto tra Poe e Mallarmé si veda Martoccia (2008).

12 "Ma occorre dir subito che tale onore d'antesignano della «Poesia pura» spetta al Poe soltanto per un equivoco. Lungi dall'aver dato i primi esempi di composizione mancanti di ogni contenuto discorsivo o concettuale e racchiudenti tuttavia la possibilità d'infiniti significati suggeriti dalla cellula musicale del verso (ché tale è il senso che da Mallarmé in poi ha il termine di «poesia pura»), il Poe subordina la piena intelligenza d'una poesia all'immedesimarsi con la situazione reale e coi sentimenti che esprime" (Poe e Praz 2012: 107). 


\section{LE IDEE SULLA TRADUZIONE DI PRAZ E LA PHILOSOPHY OF COMPOSITION}

La traduzione del Corvo probabilmente doveva fornire a Praz un'ottima palestra nella quale mettere a punto la sua metodologia e le sue idee sulla tradizione, e in particolare sulla traduzione in versi di un testo poetico. $\grave{\mathrm{E}}$ infatti noto che il testo di Poe fu accompagnato fin quasi da subito ${ }^{13}$ da un testo critico-teorico, la Philosophy of composition, in cui Poe, un poco per celia un poco seriamente, viviseziona il proprio testo analizzandone razionalmente ogni aspetto contenutistico e tecnico-stilistico, per spiegare come esso sia divenuto il testo finale.

Questa forte limitazione autoriale nell'interpretazione del testo doveva rappresentare una grossa sfida alle idee traduttorie di Praz, convinto sostenitore del bisogno che il testo tradotto non venga a discostarsi, se non nelle inevitabili differenze che comportano le diversità delle due lingue fatte entrare a contatto, dal testo originale. Si rende dunque necessario tentare di ricostruire le linee generali del pensiero sulla traduzione del critico romano.

Innanzitutto Praz ritiene la traduzione in versi della poesia non solo possibile, ma necessaria per il mantenimento del valore poetico dell'opera di partenza ${ }^{14}$. Il motivo di questa dichiarazione risiede nella concezione della poesia del critico, convinto sostenitore del fatto che una poesia ridotta al solo contenuto, privata delle proprie peculiarità ritmico-stilistiche, altro non sia che una riduzione prosastica ${ }^{15}$; concezione che comporta per necessità estetica, quasi ontologicamente legata all'essenza stessa della poesia, la resa in versi nella lingua di arrivo dell'opera di partenza ${ }^{16}$.

Questa strenua difesa della resa traduttoria in versi però non comporta assolutamente una resa libera, sottomessa all'arbitrio del traduttore. Praz sostiene infatti che si debba certo tradurre in versi, ma che sia necessario farlo con il sostegno di una puntuale conoscenza filologica, storica, psicologica del testo originale per non tradirne il messaggio e gli intenti. Ne consegue che il traduttore debba essere "prima di tutto un critico e un interprete: ma un critico il quale invece di dire spiegatamente come egli costruisca la situazione spirituale del suo autore, lo dice rifacendo sé a immagine e similitudine di

${ }^{13}$ Il corvo esce per la prima volta nel 1845 per The American review; la Philosophy of compostion l'anno successivo, cfr. Nota 11.

14 “Ogni versione in prosa di un'opera poetica è già fallita in partenza, perché la logica del discorso corrente è diversa da quella del fantasma poetico" (Praz 1956: 15).

15 "I concetti, le immagini di una poesia non hanno un valore a sé stante, indipendente dall'alone di melodia onde son circonfusi" (Praz 1925: 8).

16 "Si comprende allora la ragione della traduzione in versi, ragione estetica, intima e non ragione pratica, dettata da criteri estrinseci di mimetismo" (Praz 1925: 8). 
quell'autore stesso, rivivendo appunto quella situazione fantasticamente invece che dialetticamente" (Praz 1925: 7). Dunque il traduttore di poesia dovrà ricalcare, per quanto possibile, il metro dell'originale, specie nei testi adottanti una forma chiusa, poiché questa diventa parte della ragione estetica e della capacità suggestiva dell'opera; e questa necessità si vedrà ben soddisfatta nelle traduzioni messe in atto da Praz, che sempre strizzano l'occhio alla forma dell'originale.

Praz parla esplicitamente anche delle scelte linguistiche del suo modo di tradurre: egli, nell'Introduzione a Poeti inglesi dell'800 afferma di aver, per quanto gli sia stato possibile ${ }^{17}$, sempre operato una serie di scelte volte a ricalcare le soluzioni stilistico-linguistiche dell'originale ${ }^{18}$; tutto ciò con l'intento di offrire alla cultura di arrivo un prodotto certo traslato, ma quanto più possibile corrispondente alla posizione che questo doveva rivestire nella cultura di partenza.

Questa metodologia del tradurre detta sicuramente alcune linee guida fondamentali, ma non pone alcuna prescrizione vincolante: per Praz la teoria è valida solo se poi permette di approcciare efficacemente il testo da tradurre ${ }^{19}$.

Dunque, l'idea di traduzione di Praz sembra essere eminentemente quella di un critico, non di un poeta che potrebbe tendere alla rielaborazione del testo originale; egli sembra delineare una serie di procedure traduttive che tendono a un sistema source oriented che però tenga presente la necessità di ben far acclimatare l'opera di partenza nella cultura di arrivo, operazione questa fondata sull'individuazione e la resa quanto più fedele possibile della dominante (o delle dominanti) che costituisce, filologicamente, storicamente, psicologicamente il motivo d'esistenza del testo di partenza nella sua cultura nativa; Praz cerca nelle sue traduzioni di individuare le caratteristiche estetiche e/o informative che rendono l'opera di partenza importante nella cultura da cui deriva e di trasmetterli, adattati ma non variati nella loro essenza, alla cultura d'arrivo.

Si capisce ora molto bene quanto la Philosophy of composition dovesse risultare al contempo un punto di riferimento e un forte limitatore alla libertà nella resa in italiano del Corvo, dato che Poe in questo saggio sembra individuare esattamente le dominanti del proprio testo, in un certo senso obbligando Praz a seguire la sua linea interpretativa. In questo saggio

17 “Certo, la differenza tra quegli autori sarà ben lontana dall'essere, in italiano, quale in inglese, perché la mia individualità non poteva non interferire [...]” (Praz 1925: 7).

18 "Il Coleridge, per esempio usa arcaismi [...] non si meravigli dunque il lettore di fronte a parole inusitate" (Praz 1925: 7).

19 "In sede teoretica si può ragionar quanto si vuole sull'argomento tradurre; ma di fatto ciò che importa non è il tradurre, ma la traduzione, questa traduzione" (Praz 1925: 10). 
del 1846 infatti l'autore americano mette in luce passo passo gli strumenti utilizzati per comporre le proprie poesie, lamentando la cattiva abitudine di tanti suoi colleghi di non sapere o non volere, per malcelato narcisismo, ricostruire razionalmente i processi e le scelte che hanno informato le loro opere.

Prima di riassumere i contenuti del saggio utili ai fini di questo elaborato $^{20}$, andrà però certamente detto che molti ritennero questo testo dell'americano un mero divertissement letterario, per non sminuire il lavoro poetico che dovrebbe essere libero da tecnicismi eccessivi e guidato da ispirazione quasi divina; mentre altri, tra i quali un giovane Mallarmé, videro in esso un testo programmatico della poetica dell'americano (Poe e Praz 2012: 111-112). Praz sembra non prendere una posizione netta sulla serietà critica del saggio di Poe, limitandosi a dire che la presenza o meno del calcolo non sminuirebbe nulla della poesia dell'autore di Boston; e prosegue anzi dicendo che semmai di calcolo e di raffinazione stilistica nei versi del Poe ve ne è troppo poco, appagandosi egli di raggiungere un effetto sicuro anche se formalmente poco curato (Poe e Praz 2012: 113); si unisce così al giudizio che Huxley dà su Poe, quello di un "gentiluomo per natura, disgraziatamente inquinato da un cattivo gusto incorreggibile poiché la sostanza di Poe è raffinata, è la sua forma che è volgare"21; volgarità che lo stesso Praz riconosce individuandone la causa prima nella volontà del Poe di far poesia seguendo una strettissima logica meccanica per i ritmi e la costruzione strofica de Il corvo (v. Poe e Praz 2012: 113).

Ad ogni modo Praz dimostrerà, come vedremo nell'analisi dettagliata del testo, di tenere ben presente questo scritto per far aderire quanto più possibile la propria traduzione agli intenti dell' originale, seguendo così la propria idea che la traduzione debba rendere sempre, per quanto possibile, gli intenti e il contesto di nascita del suo originale; e sarà forse per questo che anche la traduzione di Praz si ammanta di una sfarzosissima ma trita veste poetica, intesa nella sua accezione più deteriore e ironica ${ }^{22}$.

Nel saggio Poe inizia dicendo che, per necessità legate a contingenze materiali, il Corvo doveva nascere come un prodotto in grado d'incontrare sì il favore della critica ma che al contempo dovesse arrivare a larghi strati di pubblico. Poe passa quindi a delucidare tutta la serie di scelte formali

${ }^{20}$ Per questa analisi si analizzeranno soltanto gli aspetti del saggio che Praz sembra aver tenuto in considerazione nella sua traduzione. Per la lettura integrale del testo rimando alla traduzione di Ludovica Koch (1986) o alle numerose edizioni dell'originale, reperibile anche online.

${ }^{21}$ Citato in Poe e Praz (2012: 116-117).

22 Praz rimprovera infatti a Poe "l'insistenza su certi motivi e clichés carichi di quel che volgarmente si chiama «contenuto poetico»" (Poe e Praz 2012: 113). 
da lui effettuate, partendo dalla lunghezza; su questa egli dice, seguendo Coleridge (Poe e Praz 2012: 107), che il poema, per essere interamente poesia e non alternare alle accensioni liriche momenti prosastici, deve essere necessariamente tanto breve da consentire un'unica seduta di lettura: da qui la scelta dei 108 versi del Corvo.

Segue la riflessione sull'effetto da dover suscitare nel lettore e questa riflessione trova nella Bellezza, unico campo esclusivo della poesia per l'americano ${ }^{23}$, l'emozione da dover evocare nel lettore. Una volta scelta la bellezza come effetto, Poe deve trovare il registro più adatto ad esprimerla; perciò dopo una breve riflessione giunge a notare che la bellezza quando si manifesta ai suoi più alti apici stimola sempre a malinconiche lacrime gli spiriti sensibili; da ciò deriva che il registro che più le si adegua è un registro di tristezza, e che la malinconia è il modus exprimendi più adeguato al bello e perciò alla poesia.

Capita dunque la lunghezza, l'effetto e il registro che il testo avrebbe dovuto avere, Poe passa a indagare con quali strumenti tecnici egli avrebbe meglio potuto raggiungere gli obiettivi prefissatisi.

Lo strumento più immediato che Poe rintraccia è quello del ritornello o refrain, che suscita un piacere universale perché con la sua caratteristica, la monotonia, esso comunica al lettore una piacevole sensazione di percezione di unità; ma Poe non si accontenta di ciò: egli decide di mantenere il ritornello fisso ma di cambiarne il senso posizionandolo in diversi contesti, accentuandone così ancor di più la ripetitività, poiché l'uso variato della stessa parola non fa altro che sottolineare il fatto che la parola utilizzata sia sempre la stessa (Poe e Praz 2012: 86). La scelta del refrain comporta inoltre di necessità la divisione strofica. Per variarlo così spesso egli ha chiaramente bisogno di un ritornello che possa facilmente essere adattato a contesti testuali diversi, quindi lo sceglie di una sola parola; parola che si rivela essere, quasi necessariamente, nevermore, poiché egli voleva una parola che "dovesse risultare sonora e suscettibile di un' enfasi protratta"(Poe e Praz 2012: 86) e la parola sopra vista ha in sé sia la $o$ lunga (per Poe la più sonora delle vocali) che la $r$ (la più prolungabile delle consonanti), oltre ad esprimere pienamente la malinconia che l'autore si era prefissato come registro. Scelta la parola, all'americano si poneva il problema di coniugare la ripetizione di questa con la credibilità della storia: dato che un uomo dotato di razionalità non avrebbe mai ripetuto solo e soltanto quella parola, ecco la scelta di farla pronunciare prima ad un pappagallo, poi mutato in un corvo perché molto più in linea con l'atmosfera del testo.

23 "Il piacere allo stesso tempo più intenso, più esaltante e più puro credo che lo si trovi nella contemplazione del bello" (Poe e Praz 2012: 85-86). 
A questo punto Poe decide di parlare della metrica del testo, iniziando con una requisitoria verso i colleghi poeti colpevoli, a suo dire, di non aver mai sfruttato le infinite possibilità delle combinazioni di varietà metriche e strofiche per ottenere una struttura metrica che fosse originale, individuando il motivo di questa negligenza nel fatto che per esserci originalità non ci vuole intuizione o impulso ma attenta e razionale ricerca. Egli dice dunque originale non tanto il ritmo o il metro del Corvo ("il ritmo è trocaico e il metro una tetrapodia acatalettica alternata a un eptametro catalettico, ripreso nel ritornello del quinto verso e chiuso da un tetrametro catalettico" (Poe e Praz 2012, 90)), ma ritiene originale la composizione della strofa fatta utilizzando questo ritmo e questo metro; ciò porta ad avere un'inedita composizione strofica in cui il piede utilizzato è il trocheo e che vede il primo verso di ogni strofa "composto da otto di questi piedi; sette e mezzo il secondo verso; otto il terzo; sette e mezzo il quarto e il quinto; tre e mezzo il sesto" (Poe e Praz 2012, 90) (si capisce ora il rimprovero di pedanteria matematica mosso da Praz alla poesia di Poe, per il quale cita un passo di Orfismo della parola di Francesco Flora ${ }^{24}$ ). A questa struttura metrica Poe dice di aver applicato inoltre un uso estensivo della rima e dell'allitterazione, che in effetti danno al testo una notevole uniformità fonica.

Da questo breve riassunto si possono ricavare tutta una serie di nodi dall'autore ritenuti centrali nella sua poesia, vale a dire: l'importanza della forma chiusa e delle rime unite all'allitterazione, che comportano un ritmo e una sonorità spesso ossessivamente ritornanti su loro stessi, in coerenza con il continuo ritorno all'amata Lenore dell'amante protagonista del testo; la scelta tematico-contenutistica, centrata sulla bellezza, cosa che implica un registro linguistico-stilistico di malinconia, la qual cosa si materializza in un linguaggio arcaizzante, ampolloso e, in un certo senso, da noi oggi percepito come fastidiosamente poetico ${ }^{25}$.

Vedremo ora come Praz ha cercato di rendere quanto appena visto nella sua traduzione del poema.

\section{LA TRADUZIONE DEL CORVO DI MARIO PRAZ}

La traduzione del Corvo di Mario Praz esce per la prima volta nel 1921 per Rivista d'Italia. In essa Praz sembra far reagire tutte le sue teorie sulla traduzione che nel paragrafo 2 si è tentato di sintetizzare. Praz infatti deci-

24 "Servirsi del potere ragionativo per far poesia, significa voler suonare il violino coi guantoni della boxe" citato in Poe e Praz (2012: 113).

25 “'...] d'immagini poetiche come quelle ora elencate egli si serve come di tessere musive, e ne stipa i suoi versi, in modo da influire sul lettore con un procedimento che ha qualcosa della tecnica pubblicitaria nella sua insistenza” (Poe e Praz 2012: 108). 
de di provare a rendere l'atmosfera del testo originale mantenendone con quanta più attenzione possibile gli aspetti metrici, stilistici, fonico-ritmici e linguistici. Dalla volontà di far acclimatare il testo nella cultura di arrivo, rendendone i caratteri che ne costituiscono la posizione peculiare che esso riveste nella cultura di partenza, derivano infatti le scelte (conservative nei confronti dell'originale) di mantenere la forma chiusa con il suo schema rimico e di richiamare quanto più possibile la tessitura fonica del testo inglese; allo stesso scopo sembra concorrere l'intento di ricreare, con scelte lessicali e sintattiche arcaizzanti e strizzanti l'occhio alla tradizione poetica italiana più antica, la suggestione che Poe vuole esercitare sul lettore tramite "l'insistenza su certi motivi e clichés carichi di quel che volgarmente si chiama contenuto poetico" (Poe e Praz 2012: 107).

Il tentativo di adesione al testo originale più evidente è certamente il mantenimento della rima in -ore, tanto centrale nella riflessione che Poe fa sul suo testo, con il perfetto corrispettivo italiano in -ora, pur essendo Praz ben consapevole dell'assenza di un termine in -ora esattamente corrispondente nel senso al nevermore inglese. Per questo Praz dice di aver cercato di "arieggiare il ritmo e il suono dell' originale anche a costo di un espediente sforzato, il "mai più ora" e dice di non essersi fatto scrupolo d'aver tradito anziché tradotto un capolavoro" (Poe e Praz 2012: 122), pur di riuscire a trasmettere al pubblico italiano le intenzioni poetiche tanto attentamente espresse da Poe nella Philosophy of composition.

Passeremo ora ad analizzare nel dettaglio alcune delle scelte particolari che compongono il sistema generale qui sopra esposto.

\subsection{La metrica}

Innanzitutto, la traduzione è in versi; Praz inoltre decide di trasporre il preciso e apparentemente complesso sistema metrico del testo origina$1 \mathrm{e}^{26}$ mantenendo identica la struttura strofica e adattando quella versale al sistema metrico italiano tramite la resa della struttura quantitativa del testo di partenza (che ha quattro piedi, quindi otto sillabe, per ogni emistichio) con l'ottonario italiano, raddoppiato nei primi cinque versi di ogni strofa e ripetuto solo una volta nell'ultimo verso di ognuna di esse, che ospita il ritornello; in questo modo egli replica perfettamente la quantità sillabica dei versi di Poe (che sono, per i primi cinque versi di ogni strofa, dei tetrametri trocaici acatalettici alternati a degli eptametri catalettici, aventi quindi rispettivamente sedici e quindici sillabe, chiusi da un tetrametro catelettico che ha sette sillabe). Questa scelta inoltre sembra in perfetta coerenza con l'origine supposta dell'ottonario italiano, che per D'Ovidio deriverebbe dall'emi-

\footnotetext{
${ }^{26}$ Cfr. Paragrafo 3.
} 
stichio dell'ottonario trocaico resosi autonomo ${ }^{27}$, ottonario trocaico che è l'esatto corrispondente latino del metro utilizzato da Poe nel suo poema.

Riporterò ora il primo verso e il secondo della prima strofa in inglese e in traduzione in modo da esemplificare quanto appena detto:

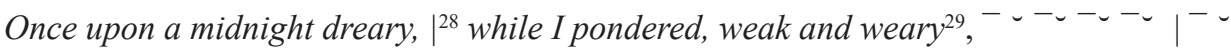
-

Over many a quaint and curious $\mid$ volume of forgotten lore

Una mezzanotte grave, | meditavo affranto e frale $1357 \mid 357$ su volumi strani e rari |di dottrina ch'or s'ignora: $357 \mid 357$

Visto poi lo spazio dato da Poe nella Philosophy alla riflessione sull'importanza del ritornello, all'accurata scelta fonica effettuata per giungere al rimante in -ore (Poe e Praz 2012: 87), alla sottolineatura dell'"uso estensivo della rima e dell'allitterazione" (Poe e Praz 2012: 91), l'Illustre Anglista decide di mantenere lo schema rimico dell'originale che è così schematizzabile: (a) $a b$ (c) $c$ (c) $b b b$. Va da sé che uno schema così ossessivo e ricco di rime interne non è facilmente trasponibile in un'altra lingua (specie se quest'ultima ha una fonetica sostanzialmente diversa come è il caso dell'italiano nei confronti dell'inglese) mantenendo al contempo il pieno senso del testo; ma Praz ci riesce in quasi tutte le occasioni, pagando a volte il piccolo dazio della sostituzione di una rima perfetta con una rima imperfetta (egli utilizza per lo più l'assonanza in sostituzione di alcune rime interne non replicabili); ciò si può ben vedere dal precedentemente riportato esempio, dove la rima perfetta dreary : weary è resa con l'assonanza tra grave e frale; varrà la pena, sebbene gli esempi di questo stratagemma siano molto presenti nel testo, riportarne almeno un altro esempio tramite la resa traduttoria di $\mathrm{I}, 3^{30}$ :

While I nodded, nearly napping, suddenly there came a tapping, $\rightarrow$ in sopor quasi ero assorto, quando giunse un sùbito urto,

27 "E propriamente un emistichio del tetrametro trocaico acatalettico, cioè a chiusa non cretica o sdrucciola, e perciò detto dai latini ottonario trocaico" (D'Ovidio 1932: 224).

${ }^{28}$ In questo come in altri casi seguenti il simbolo | è utilizzato per indicare la cesura tra i due emistichi versali.

${ }^{29}$ Sia il testo inglese che la traduzione sono in Poe e Praz (2012). Per quanto riguarda le citazioni dei versi di entrambi i testi si è scelto di usare il corsivo per segnalare sia quelle del testo inglese che quelle della traduzione.

${ }^{30}$ Quando verranno citati versi dei due testi si è scelto per comodità di indicarli numerando la strofa con numero romano e il verso con numero arabo, mancando nell'edizione di riferimento una numerazione progressiva dei versi. 
particolarmente interessante poiché la rima interna napping : tapping viene mantenuta grazie all'utilizzo di un istituto trecentesco qual è la rima siciliana, presente tra le parole assorto : urto; rima siciliana che torna anche nella terza strofa (paura, v. 2 : implora, v. 4; ancora, v. 6) e nell'ottava (positura, v. 2 assona con ombra, vv. 4 e 5 e rima con ora, v. 6).

Praz inoltre sembra voler richiamare l'accentuazione fissa del metro trocaico utilizzando quanto più possibile l'ottonario "classico", con accenti principali sulla terza e sulla settima sillaba ${ }^{31}$, che coprono il $78 \%$ dei versi totali; inoltre, all'interno degli ottonari accentati in terza e in settima sede spiccano quelli con ictus di $3^{\mathrm{a}}-5^{\mathrm{a}}-7^{\mathrm{a}}(35 \%)$ e quelli con accenti principali di $1^{a}-3^{a}-5^{a}-7^{a}(30 \%)$ a conferma del tentativo di emulare quanto più possibile la musicalità fissa del testo originale. A titolo di esempio riporto la scansione metrica della prima strofa:

Una mezzanotte grave, | meditavo affranto e frale 1 (3) $57 \mid 357$

su volumi strani e rari $\mid$ di dottrina ch'or s'ignora: $357 \mid 357$

in sopor quasi ero assorto, | quando giunse un sùbito urto, $357^{32} \mid 1357$

come s'un picchiasse sordo |l'uscio della mia dimora. $1357 \mid 1357$

Mormorai: «sarà qualch'ospite, |ch'urta l'uscio alla dimora: $357 \mid 137$

questo sol: null'altro ancora. 1357

Mi pare infine interessante notare che l'ottonario di $3^{\mathrm{a}}-7^{\mathrm{a}}$ (e per lo più quello, in un certo senso più ripetitivo e pesante, con accentazione di $1^{\text {a }}$ $\left.3^{a}-5^{a}-7^{a}\right)$ sia sempre usato nel luogo più in vista delle strofe, vale a dire la chiusa mono-ottonaria che ospita il ritornello; certo Praz è in questa resa aiutato dal fatto che un terzo di questi versi sono identici ${ }^{33}$ (in inglese quoth the raven, «nevermore» reso in italiano con Disse il Corvo: "Mai più, ora»):

I, 6: questo sol: null'altro ancora. 1357

II, 6: senza nome qui da allora. 1357

IV, 6: ombra, là; null'altro ancora. 1357

V, 6: solo, e poi più nulla ancora. 1357

VI, 6: vento egli è, null'altro ancora». 1357

VII, 6: si posò, null'altro ancora. 357

VIII, 6: Disse il Corvo: «Mai più,ora» 1357

${ }^{31}$ Beltrami (2011: 197).

${ }^{32} \mathrm{Si}$ è preferito qui contare come accento principale quello di $5^{\mathrm{a}}$ piuttosto che quello di 4 a . Infatti "quasi" sintatticamente ha più rilevanza e potrebbe perciò essere ritenuto a ragione un miglior foriero di ictus rispetto a "ero". Ma l'evidente inerzia ritmica della strofa ha fatto preferire una lettura con accento principale di $5^{\mathrm{a}} \mathrm{su}$ "ero".

${ }^{33}$ Inserisco di seguito la scansione dei versi chiudenti ogni strofa, omettendo i versi identici qualora si ripetano. 
IX, 6: con tal nome: «Mai più, ora». 357

XI, 6: "Non più mai, giammai più, ora"». 357

XII, 6: dir volea con «Mai più, ora». 1357

XIII, 6: Lei non preme mai più, ora! 1357

XVIII, 6: non risorge più da allora! 357

La scansione dei versi è stata effettuata tenendo conto dell'evidente inerzia ritmica del testo: si sono perciò considerati accenti principali alcuni accenti dubbi (ad esempio, il $3^{\circ}$ in $\mathrm{V}, 6$ ) se in $1^{\mathrm{a}}, 3^{\mathrm{a}}, 5^{\mathrm{a}}, \mathrm{o} 7^{\mathrm{a}}$ posizione; per lo stesso motivo, e per evitare un continuo contraccento di $6^{\mathrm{a}}-7^{\mathrm{a}}$, si è scelto di considerare come accento principale quello di $5^{\mathrm{a}}$ in tutti i casi in cui sono a contatto le parole mai e più, entrambe possibili portatrici di ictus (cfr. v. 6 delle strofi dall'VIII alla XVII). È certo possibile considerare il contraccento di $6^{\mathrm{a}}-7^{\mathrm{a}}$, soprattutto vista la dialefe tra più ed ora presente nei versi sopra indicati. Questa lettura, per quanto corretta, mi pare però poco in linea con le idee sulla traduzione di Praz, che ipotizzo abbia preferito questa scansione cantilenante e senza increspatura contraccentuativa per rendere l'andamento ritmicamente fisso del testo originale, in modo da rendere "la vitalità fissa, allucinata" (Poe e Praz 2012: 113) che egli ritiene abbia la poesia di Poe ${ }^{34}$. Una traduzione che tenga conto in questa maniera di fattori esterni al solo contenuto di certo non può essere letterale. E se abbiamo già visto come anche lo stesso Praz $^{35}$ fosse consapevole della necessità di tradire in alcuni luoghi la lettera del testo inglese (unico modo per non tradirla in nulla sarebbe stata una traduzione di servizio in prosa, inammissibile nell'ottica del critico romano) non si è ancora notato come ciò si rifletta nel rapporto tra il metro e la sintassi. Infatti risultano nel testo italiano alcune inarcature in più rispetto a quelle presenti nell'originale, e Praz sembra in questo seguire una tendenza tipica della traduzione italiana, che, forse per una propensione maggiore all'enjambement insita alla propria tradizione poetica, tende a restituire testi tradotti molto meno rispettosi del rapporto uno a uno tra il verso e la frase presenti nei testi modello. A ciò andrà poi aggiunto il problema della naturale necessità di riorganizzare il rapporto metro-sintassi, inevitabile quando in traduzione si decide di mantenere la misura versale e le varie rime. Alcuni esempi ben chiariranno quanto finora detto:

${ }^{34}$ Questa interpretazione sembra inoltre confortata anche dalle parole di Poe che in un altro suo saggio teorico, The rationale of verse, dove l'americano dice esplicitamente che uno stesso andamento ritmico protratto lungo il testo può portare a scandire con lo stesso ritmo versi altrimenti leggibili in maniera diversa (Il testo originale è reperibile online al seguente link: https://web.english.upenn.edu/ cavitch/pdf-library/Poe_Rationale.pdf; manca invece una traduzione italiana affidabile).

${ }^{35}$ Cfr. Paragrafo 2. 
II, 1-3: Ah, distinctly I remember it was in the bleak December. / And each separate dying ember wrought its ghost upon the floor. / Eagerly I wished the morrow; - vainly I had sought to borrow $\rightarrow$ Fu in dicembre, mi rammento, e ciascun tizzo morendo / movea sopra il pavimento il suo spettro, ed io l'aurora / col desio bramavo solo, poi ch'invan cercai consolo.

Qui 1'originale non presenta alcuna inarcatura; Praz invece traduce inserendo due inarcature tra il soggetto e il verbo principale tra i vv. 1-2 e ancora tra i vv. 2-3; si nota inoltre una maggior tendenza all'ipotassi di contro alla scelta giustappositiva dell'originale (v. 3: la coordinata vainly I had sought to borrow è trasformata nella causale poi ch'invan cercai consolo); infine è evidente il turbamento dell'ordine naturale dei costituenti al v. 2 (il complemento oggetto è allontanato dal verbo con l'inserimento del complemento di luogo).

XI, vv. 1-2: Startled at the stillness broken by reply so aptly spoken, I

"Doubtless," said I, "what it utters is its only stock and store $\rightarrow$ Stupefatto per tal botta e risposta, ch'avea rotta / la quiete: «Ogni sua merce è quel ch'egli mette fuora,

Qua l'anticipazione del complemento di causa obbliga la traduzione italiana ad aggiungere un'inarcatura tra verbo e oggetto della subordinata completiva.

XII, vv. 4-5: Fancy unto fancy, thinking what this ominous bird of yore- / What this grim, ungainly, ghastly, gaunt, and ominous bird of yore $\rightarrow$ meditai qual malaugurio quell'augel dei tempi ch'ora / non son più, lo strambo e tristo e sinistro augel d'allora.

Nell'originale l'utilizzo di yore permette a Poe di terminare la sua frase in concordanza con la fine del verso, Praz invece utilizzando il sintagma dei tempi ch 'ora non son più è "costretto" a distribuirlo tra i due versi inarcando tra questi la relativa, oltre a variare l'originale che ripete yore.

XIV, vv. 1-3: Then, methought, the air grew denser, perfumed from an unseen censer / Swung by Seraphim whose foot-falls tinkled on the tufted floor. / "Wretch," I cried, "thy God hath lent thee-by these angels he hath sent thee $\rightarrow$ L'aer parve allor più denso, quasi fosse infuso incenso / invisibil da seràfi de'cui passi era sonora / la mia stanza. Ed io: "Dolente! Con quegli angeli Iddio scende.

L'anticipazione di infuso nel testo italiano crea un'inarcatura tra il sintagma nominale formato da incenso e il suo aggettivo invisibil; inoltre, nel testo inglese non c'è enjambement tra i versi 2 e 3 mentre una rottura è presente nei versi italiani, che vedono la frattura forte tra il verbo era sonora e il suo soggetto la mia stanza. 
Ad un principio simile risponde la tendenza del testo italiano ad aumentare le cesure tra i due emistichi non coincidenti con i limiti sintattici di questi, cosa che serve inoltre a rendere meno macchinalmente scandita la versificazione del poeta americano. Ne sono alcuni esempi, tra i molti possibili:

IV, 3: But the fact is I was napping, | and so gently you came rapping, $\rightarrow$ ma sopito m'ero quando | siete giunto voi picchiando dove la cesura cade all'interno della subordinata temporale, tra la congiunzione e il verbo di essa;

VIII, 2: By the grave and stern decorum | of the countenance it wore, $\rightarrow$ tanto grave il suo sussiego | era, in quella positura. Qui la cesura cade tra il soggetto e il verbo, separando oltretutto tra i due emistichi la copula e il nome del predicato;

IX, 2: Though its answer little meaning | -little relevancy bore; $\rightarrow$ ben che irrilevante e senza | senso avesse la parola: dove ad essere rotto dalla cesura è il sintagma aggettivale senza senso, interrotto tra la preposizione e il sostantivo;

$\mathrm{X}, 1:$ But the Raven, sitting lonely $\mid$ on that placid bust, spoke only $\rightarrow M a$ posando sol sul placido | busto, il Corvo, quasi l'animo dove ancora una volta è un sintagma nominale composto da aggettivo e sostantivo a cadere a cavaliere dei due emistichi;

XIII, 2: To the fowl whose fiery eyes now | burned into my bosom's core; $\rightarrow$ i cui fiammei occhi in fondo | al mio petto ardean tuttora; dove il complemento di stato in luogo, in fondo al mio petto, si trova interrotta dalla pausa dei due emistichi.

\subsection{Il contenuto "volgarmente poetico": tratti arcaizzanti nel lessico e nella morfosintassi nella traduzione praziana}

Come detto nell'introduzione di questo terzo paragrafo, Praz mette in moto tutta una serie di stratagemmi per cercare di rendere il linguaggio arcaizzante di Poe nella sua traduzione italiana. Va detto che, essendo l'italiano una lingua con una tradizione poetica di alcuni secoli più antica $\mathrm{e}$ avendo una lingua letteraria molto più conservativa rispetto all'inglese, le frecce alle quali Praz può attingere dalla sua faretra sono numerose e molto stratificate nel tempo (andando dalla poesia delle origini fin quasi alla sua stessa epoca). Quanto appena detto comporta che spesso Praz si trovi ad innalzare ancora di più la temperatura stilistica già alta del testo originale; questo per far sì che il contesto italiano, ben più abituato all'innalzamento 
di tono del discorso poetico di quello inglese, recepisca quanto i versi di Poe dovessero suonare arcaici e aderenti alla tradizione all'orecchio di un inglese di metà ottocento.

Da un punto di vista molto generale a questo innalzamento e arcaizzazione del dettato concorrono innanzitutto le numerosissime apocopi (ce ne sono svariati esempi lungo tutto il testo; per economia si citeranno solo quelle presenti nella prima strofa: or, v. 2; sopor, v. 3; s'un, v. 4; qualch'ospite, v. 5 ; sol, v. 6); sempre in questa linea generalmente arcaizzante s'iscrivono altri fenomeni morfologici come l'utilizzo del pronome personale di terza persona ei in luogo di egli, e a maggior ragione di lui (VII, 3 ei non fece alcun saluto; XI, 4 quei lai ch'ei disse), e la forma dell'imperfetto senza fricativa labiodentale sonora (II, 2: movea; XI, 4: avean; XII, 1 sorridea; XII, 6 volea), utilizzi questi che sono utili anche a mantenere il computo sillabico dei versi.

Numerosissime sono le scelte lessicali effettuate pescando da un registro linguistico che all'altezza del 1921 era già sentito come desueto. Si riportano solo alcuni significativi esempi:

I, 1: frale è allotropo letterario del più comune fragile, utile al contempo al computo sillabico e all'assonanza interna con il precedente grave;

I, 4; chamber viene reso sineddoticamente con dimora, mantenendo la sfumatura non comune di chamber ${ }^{36}$ e la fondamentale rima in -ora;

II, 3: eagerly I wished viene reso con col desio bramavo dove sia la preposizione articolata agglutinata, sia l'allotropo letterario desio e l'uso di bramavo innalzano il tono rispetto all'originale; al verso 4 sempre della seconda strofa surcease of sorrow viene reso con tregua al duolo, e qui l'allotropo letterario duolo sembra coerente con l'altrettanto arcaico surcease, in una sorta di compensazione;

III, 1: serico è certo arcaico ma al contempo è anche il corrispettivo perfetto del silken inglese e la terna serico, vago, mesto (per l'originale silken, sad, uncertain) richiama facilmente alla memoria del lettore italiano echi petrarcheschi;

IV, 2: venia per rendere forgiveness ha valore arcaizzante; al verso 4 della stessa strofa fuora (forma arcaica e poetica di fuori) è usato in maniera del tutto originale rispetto all'originale verso inglese (And so faintly you came

${ }^{36}$ Chamber nel senso di camera da letto appartiene alla lingua arcaica come indicato nell'Oxford English Dictionary. Per verificare frequenza e arcaicità delle parole usate da Poe in lingua inglese si è fatto riferimento alle rispettive voci dell'opera vocabolariale suddetta. 
tapping, tapping at my chamber door,) probabilmente per mantenere la sempre fondamentale rima in -ora;

$\mathrm{V}, 1$ : darkness è reso con tenebrore, termine poetico e arcaizzante utile alla rima interna con timore, e tutto questo verso viene in realtà rielaborato ai fini della resa della rima interna originale (Deep into that darkness peering, long I stood there wondering, fearing $\rightarrow$ Gli occhi per quel tenebrore spinsi in dubbio ed in timore; è facilmente visibile il cambiamento di soggetto dall'I inglese a gli occhi praziani);

VI, 1: qua si vede nel secondo emistichio una frase nominale estremamente letteraria (pieno il cuore di doglianza) dove l'anastrofe lieve sembra quasi sottolineare la rima in -anza (che accende ricordi addirittura provenzali per l'utilizzo del sostantivo suffissale) ottenuta con l'utilizzo di un altro allotropo letterario; ciò rende un secondo emistichio molto più "comune" dal punto di vista lessicale di Poe: all my soul within me burning, sebbene anche in esso si possa osservare l'anastrofe di within me tra soggetto e verbo;

VII, 2: lo yore di partenza, letterario, è reso altrettanto letterariamente con il sintagma lungi d'ora.

È però l'VIII strofa a rappresentare il parossismo letterario di entrambi i testi, probabilmente per il carattere ironico che le assegna l'esigenza narrativa: in questa strofa infatti il Corvo entra nella stanza e viene visto per la prima volta dall'Amante, che in un primo momento si fa beffe della figura grave dell'uccello. Nell'originale si ha infatti tutta una serie di parole arcaiche o letterarie come decorum, countenance, craven che, uniti all'utilizzo dell'aggettivo possessivo arcaico thy in luogo di your e alla posposizione del vocativo ("Tough thy crest be shorn and shaven, thou,»I said, "art sure no craven / ghastly grim and ancient Raven wandeing from the nightly shore -), danno un innalzamento di tono sensibile; e Praz non esita a seguire il testo di partenza utilizzando termini quali augel, negro ed egro al v.1 oltre a sussiego e positura al v. 2; va inoltre detto che il termine positura, afferendo al campo semantico dell'araldica ${ }^{37}$ sembra strizzare l'occhio all'originale $\operatorname{craven}^{38}$, poiché, pur non traducendolo direttamente, rimanda allo stesso campo semantico cui fa riferimento il gioco di parole presente nell'originale.

IX, 1: augello rende 1'altrettanto arcaico termine, atto a indicare un volatile generico, fowl; tutto il verso 3 è reso in maniera molto arcaica (For we cannot help agreeing that no living human being $\rightarrow$ convenir dobbiam

\footnotetext{
${ }^{37}$ Cfr. La voce Positura nel Grande dizionario della lingua italiana.

${ }^{38}$ Cfr. La nota 1 del traduttore in Poe e Praz (2012: 18).
} 
che niuna viva umana creatura) tramite l'utilizzo di numerose apocopi e di niuna, allotropo letterario di nessuna; al verso 5 sculto è latinismo, in luogo del più comune scolpito;

$\mathrm{X}, 3$; il sintagma non proruppe in altri lai, con lai che è termine dantesco, è usato per rendere l'inglese nothing farther than he uttered che non ha questa sfumatura letteraria.

Nell'XI strofa abbiamo invece un quasi incredibile abbassamento di tono al verso 1 nella resa di by reply so aptly spoken con con tal botta e risposta, ma Praz sembra quasi voler compensare questa necessità impostagli dal mantenimento della rima al mezzo (botta - rotta) profondendosi in due versi fortemente letterari (il 4 e il 5: sin ch'avean quei lai ch 'ei disse sol quel ritornello ancora / sin ch'ai guai di sua speranza ritornello fu, che accora) per i molti elementi poetici come il termine lai, le forme ei e avean e i numerosi troncamenti, oltre all'ordo verborum fortemente turbato, con il verbo in cluasola del verso 5.

XVI, 3: spirto è forma sincopata e letteraria di spirito.

Infine in XVIII, 1 abbiamo il termine svolando, di ascendenza pascoliana e dannunziana e molto usato nelle traduzioni da Leone Traverso (Organte 2018: 269), per il non letterario flittting.

È interessante notare inoltre come Praz alcune volte eviti le ripetizioni a stretto giro dell'originale, forse per venire incontro alla maggiore tendenza alla variatio tipica della lingua italiana (I, 4: As of some one gently rapping, rapping at my chamber door. $\rightarrow$ come s'un picchiasse sordo l'uscio della mia dimora.; II, 4: From my books surcease of sorrow-sorrow for the lost Lenore- $\rightarrow$ tra i miei libri, tregua al duolo per la persa Leonora).

Anche sintatticamente le scelte di traduzione di Praz si orientano verso una lingua sentita come poetica e retoricamente costruita, innalzando spesso l'artificiosità del testo di partenza. A questo proposito va ancora una volta detto che nell'operazione l'Anglista è aiutato dalla maggior flessibilità di costruzione del periodo dell'italiano rispetto a quello inglese e da un'eredità poetica, quella della Penisola, più propensa al turbare l'ordine normale dei propri costituenti sintattici. Anche per questo aspetto alcuni esempi significativi, tra i molti:

III, 2: qui abbiamo un dittico di verbi riflessivi con il pronome enclitico (penetravami, assillavami di fantastica paura). È possibile giustificare la prima di queste enclisi con la legge di Tobler-Mussafia, regola tipica dell'italiano antico (Brucale 2010) e perciò contribuente ad attribuire al testo quell'aura di poeticità arcaica, trecentesca, alla quale anche altri fattori concorrono. La 
seconda enclisi non è giustificabile con la stessa legge, ed è perciò precisa scelta stilistica del traduttore che forse vuol ricalcare l'originale inglese nella posposizione del complemento oggetto al verbo (Thrilled me-filled $m e$ ); la doppia enclisi è utile poi per avere sinalefe tra i due verbi e rientrare nel computo sillabico;

simile è il discorso da fare per IV, 1 (Ecco, si fe'forza il cuore; senza indugio più: "Signore) dove la posposizione dell'avverbio più, oltre a dare una certa patina letteraria, ricalca l'originale hesitating then no longer ed evita la sinalefe tra più e indugio che avrebbe falsato il computo dell'ottonario; al verso 2 (chiedo a voi venia, credetemi, o signor,» dissi «o signora) abbiamo un innalzamento rispetto all'originale ottenuto tramite la posposizione e lo scioglimento del pronome obliquo del complemento di termine a voi rispetto all'inglese neutro truly your forgiveness I implore; al verso 3 abbiamo un'anastrofe accompagnata da una perifrasi lessicalmente connotata ( $m a$ sopito $m$ 'ero quando siete giunto voi picchiando) che ancora una volta alza la temperatura retorica rispetto all'originale I was napping, dove to nap è certo meno letterario di assopirsi;

VII, 5: il verso è turbato da un iperbato (Perched upon a bust of Pallas just above my chamber door $\rightarrow$ dove un busto era di Pallade sul portal della dimora) che mette ancor più in risalto il nome di Pallade, scelto da Poe per la sua sonorità e per sottolineare la natura intellettuale del suo protagonista (Poe e Praz 2012: 92);

XV, 5: questo verso (Di, se balsamo evvi in Gilead-dillo, dillo, ad un che implora!») vede l'utilizzo congiunto dell'avverbio di luogo più letterario $v i$ unito alla sua posposizione al verbo essere, cosa che dà un effetto arcaizzante ma che al contempo serve alla sinalefe con balsamo e perciò al computo sillabico.

Infine, anche dal punto di vista morfologico si individuano diverse scelte fatte con l'occhio volto al passato della tradizione letteraria italiana; ne sono ottimi esempi, oltre al già citato e quasi onnipresente uso delle apocopi e degli imperfetti senza fricativa labiodentale:

IV, 5: That I scarce was sure I heard you"- here I opened wide the door $\rightarrow$ che mi parve udirvi appena», - l'uscio apersi tutto, allora qui Praz utilizza la forma arcaica apersi in luogo di aprii; va però notato che in questo stesso verso il traduttore rende I scarce was sure con mi parve udirvi, dove parve è meno poetico dell'altrettanto utilizzabile parse ${ }^{39}$ per l'esigenza di

\footnotetext{
${ }^{39} \mathrm{http}$ ://www.treccani.it/vocabolario/parere2/; si rimanda inoltre alla voce parere del Grande dizionario della lingua italiana.
} 
mantenere la disseminazione fonica della sibilante presente nell'originale (scarce was sure) rendendola in italiano con quella della fricativa labiodentale (parve udirvi);

V, 4: Praz utilizza susurrare, forma meno usata del più comune sussurrare ${ }^{40}$;

IX, 1: Mi stupii d'udir parlando chiaramente augello strambo; qui possiamo notare un utilizzo del gerundio di pertinenza dell'italiano antico ${ }^{41}$, venendo questo modo utilizzato in luogo di un participio presente in quanto ha in questo verso valore relativo; questo uso sembra dipendere dalla necessità di mantenere l'assonanza interna tra parlando e strambo;

$\mathrm{X}, 3$ : l'inglese not a feather then he fluttered è reso con non fruscio uscio dall'ali e il verbo uscio con epitesi della $o$ è ancora una volta una forma arcaica; l'uso di questo verbo sembra essere adatto a rendere l'assonanza della frivativa labiodentale sorda dell'originale trasponendola in una rima inclusiva a strettissimo contatto (fruscio - uscio);

è curioso il fatto che al verso 3 e 4 della quattordicesima strofa, segnalandolo accortamente in nota, Praz utilizzi transitivamente il verbo scendere (la mia stanza. Ed io: $<<$ Dolente! Con quegli angeli Iddio scende / tregua a te, tregua e nepente ${ }^{42}$ dal pensier di Leonora), probabilmente per fare in modo di mantenere l'assonanza interna al verso tra dolente e scende; al verso 5 della stessa strofa inoltre si può osservare che l'imperativo inglese forget è tradotto, senza apparente motivo se non l'intento arcaizzante, con l'imperativo proclitico ti scorda, forma di questo modo verbale tipica della lirica italiana tradizionale ${ }^{43}$, dove viene utilizzata per accentuare il patetismo; questo utilizzo è coerente con la situazione narrativa di questa strofa dove l'amante invoca non ascoltato un balsamo capace di guarirlo dal dolore per la scomparsa Leonora;

XV, 1: l'inglese "Prophet!" said I, "thing of evil!-prophet still, if bird or devil! è reso con «O profeta» urlai «flagello! Sii tu demone od augello!

${ }^{40}$ Sussurrare in Grande dizionario della lingua italiana. La forma scempia, presente fin dalle origini, è ancora in D'Annunzio e in Manzoni, mentre Pascoli e Carducci sembrano preferire la forma geminata.

41 "In italiano antico, diversamente da quello che accade nella lingua moderna, la proposizione gerundiale poteva anche fungere da attributo a un nome, vale a dire come frase relativa" (Egerland 2010).

${ }^{42}$ Altro termine letterario, usato da D’Annunzio, Pascoli e Carducci, cfr. Nepente in Grande dizionario della lingua italiana.

${ }^{43}$ Cfr. RVF 39 in Petrarca (2018) e la canzone XXXII del libro II delle rime di Bernardo Tasso (1995). 
Dove si vede l'utilizzo di sii, che è forma arcaica della terza persona del congiuntivo presente ${ }^{44}$ e non, come potrebbe sembrare a un lettore moderno, un imperativo.

\section{L'IMPORTANZA DELLA RESA FONICA NELLA TRADUZIONE DI PRAZ}

Poe, sempre nella Philosophy of compostion (Poe e Praz 2012: 91), dice di aver fatto un uso estensivo del principio della rima e del principio dell'allitterazione. E in effetti, anche solo a una prima lettura del testo, è impossibile non rimanere stupiti di quanto ossessive siano le trame foniche del testo, accompagnate spesso da ripetizioni di interi lessemi anche a strettissimo contatto (alcuni esempi: I, 4: As of some one gently rapping, rapping at my chamber door; II, 4 From my books surcease of sorrow-sorrow for the lost Lenore) o la ripetizione in successione di versi identici o quasi (III, 4-5'Tis some visiter entreating entrance at my chamber door / Some late visiter entreating entrance at my chamber door).

Se da una parte Praz, seguendo in questo una consuetudine tipica della lingua letteraria italiana, tende a variare le ripetizioni più evidenti (traduce infatti i primi due esempi sopra riportati in questo modo: I, 4: come s'un picchiasse sordo l'uscio della mia dimora.; II, 4 tra i miei libri, tregua al duolo per la persa Leonora) dall'altra compie sforzi enormi per mantenere quanto più possibile la suggestione data dai continui echi fonici (che tanta parte gioca nel fascino immediato che suscitò il poema di Poe), non limitandosi al semplice mantenimento dello schema rimico, già di per sé di difficile resa ${ }^{45}$, ma replicando, a volte variate, molte delle tessiture foniche e delle allitterazioni del testo inglese.

Alcuni esempi, uniti ad altri riportati nei paragrafi precedenti, ben chiariranno quanto Praz dovesse considerare vitale questo aspetto:

I, 1: once upon a midnight dreary, while I pondered, weak and weary, $\rightarrow$ Una mezzanotte grave, meditavo affranto e frale. Qui Praz sembra rievocare l'allitterazione presente in weak and weary rendendola con la ripetizione del nesso fra in affranto e frale;

V, 2: Doubting, dreaming dreams no mortals ever dared to dream before; $\rightarrow$ con stupor sognando sogni quali niuno osò sinora dove l'allitterazione

${ }^{44}$ Una forma praticamente identica si ha nell'Inferno dantesco: cfr. Dante Alighieri, Inferno, I, 65-66: “«Miserere di me» gridai a lui, / «qual che tu sii, od ombra od omo certo!»”.

${ }^{45}$ Cfr. Paragrafo 3.1. 
della dentale (doubting, dreaming dreams [...] dared to dream) viene sostituita da quella della sibilante (stupor sognando sogni [...] osò sinora) cosa che spiega anche la preferenza di sinora rispetto a finora;

VII, 1: Open here I flung the shutter, when, with many a flirt and flutter, $\rightarrow$ Spalancai le imposte, quando li, sfrusciando, svolazzando, dove la dittologia allitterativa flirt and flutter viene resa in sibilante da sfrusciando, svolazzando;

VIII, 4: Ghastly grim and ancient Raven wandering from the Nightly shore $\rightarrow$ torvo corvo che migrando vieni dalla notturna Ombra. L'assonanza ghastly grim è sostituita dalla rima torvo corvo a strettissimo giro;

XI, 3: presa da un padrone triste che sciagura strinse strinse che traduce il verso 3 e parte del verso 4 dell'originale: Caught from some unhappy master whom unmerciful Disaster / Followed fast and followed faster till his songs one burden bore. Qui si può notare una delle poche deroghe di Praz all'eliminazione delle ripetizioni a stretto giro, anzi egli in questa occasione rende l'assonanza dell'originale Followed fast and followed faster trasformandola nell'allitterazione della sibilante presente in sciagura strinse strinse con una ripetizione a contatto assente nell'originale;

XII, 3: traducendo i versi 3 e 4 dell'originale (Then, upon the velvet sinking, I betook myself to linking / Fancy unto fancy, thinking what this ominous bird of yore) con e, affondando nel velluto, sogno sovra sogno aggiunto I meditai qual malaugurio quell'augel dei tempi ch'ora Praz innanzitutto ritarda ancor di più la comparsa della principale, inserendo la modale tra l'incidentale e quest'ultima. Inoltre, rende alla perfezione l'allitterazione di Fancy unto fancy con sogno sovra sogno, anzi aumentando il numero di parole allitterate;

XIV, 1: Then, methought, the air grew denser, perfumed from an unseen censer $\rightarrow$ L'aer parve allor più denso, quasi fosse infuso incenso dove la ripresa fonica originale presente in unseen censer viene resa con l'allitterazione del fono in di infuso incenso, e ripresa in più all'inizio del verso successivo con il termine invisibil;

XVIII, 2: la paronomasia dell'originale in On the pallid bust of Pallas viene resa, forse con anche maggior sottolineatura dell'originale vista la maggior vicinanza, con busto pallido di Pallade. 


\section{BIBLIOGRAFIA}

Beltrami P. G. (2011). La metrica italiana. Bologna: Il Mulino.

Brucale L. (2010). Legge Tobler-Mussafia. In Enciclopedia dell'italiano $A-L$. Roma: Treccani.

D’Ovidio, F. (1932). Versificazione romanza: poetica e poesia medievale (vol. 1). Napoli: Guida.

Egerland V. (2010). Gerundio. In Enciclopedia dell'italiano A-L. Roma: Treccani.

Mann, T. (2017). Doctor Faustus, traduzione di Luca Crescenzi. Milano: Mondadori.

Martoccia, G. (2008). Mallarmé traduttore di Poe. Annali online di Ferrara - Lettere, Vol. II, 80-120.

Organte, L. (2018). Poesia e traduzione a Firenze (1930-1950). Padova: Antenore.

Petrarca, F. (2018). Canzoniere. Milano: Mondadori.

Poe, E. A. (1986). Filosofia della composizione e altri saggi, a cura di Ludovica Koch. Napoli: Guida.

Poe, E. A. (2014). The Philosophy of Furniture. Scotts Valley: Createspace Independent Pub.

Poe, E. A. e Praz, M. (1921). Il corvo. Rivista d'Italia, 399-402.

Poe, E. A. e Praz, M. (2012). Il Corvo; filosofia della composizione / Edgar Allan Poe, traduzione a cura di Mario Praz; con illustrazioni di Gustave Dorè. Milano: SE.

Praz, M. (1925). Poeti inglesi dell'ottocento. Firenze: R. Bemporad.

Praz, M. (1956, Dicembre). Problemi del tradurre. Scuola e cultura del mondo, 1, pp. 14-19).

Praz, M. (1964). La filosofia dell'arredamento. I mutamenti del gusto della decorazione interna attraverso i secoli. Milano: Longanesi e Co.

Praz, M. (1972). Poe, genio d'esportazione. In Il patto col serpente. Paralipomeni di «La carne, la morte e il diavolo nella letteratura romantica» (pp. 41-118). Milano: Mondadori.

Praz, M. (2003). Fortuna di E. A. Poe nel primo centenario della morte. In Cronache letterarie anglosassoni (vol. II, pp. 144-148). Roma: Edizioni di Storia e Letteratura.

Tasso, B. (1995). Rime 1: I tre libri degli Amori. Torino: RES. 


\section{MARIO PRAZ TRANSLATOR OF EDGAR ALLAN POE'S THE CROW}

\section{Summary}

The article analyses one of Mario Praz's first translations from the English language. The analysis supports a metric-stylistic and historical-linguistic point of view, and results in an illustration of the translator's main methods. Edgar Allan Poe's translation of The Crow was selected for its translation-choice examples, which Praz will use in his future Italian translations. These choices, as seen through his critical works' analysis, during which the author reflects on his translation methods, are characterized by the continuous attempt to identify and transpose the dominants that constitute the main reasons of composition and the cultural position that the source text has in its own language. In his translation of The Crow, Praz decides to maintain the original metric shape as much as possible, and adapts it to the Italian metric system. Moreover, he retraces the intricate rhyming scheme and the many phonic textures of the original text. From the linguistic point of view, he makes many choices to recall Poe's archaic language. The final outcome is a text that comes close to the original text's atmosphere and transposes his charm together with some of his limits.

Keywords: Praz, translation, The Crow, Poe, dominants, stylistic. 